\title{
A Review of the Recommendations Governing Quality Assurance of Ultrasound Systems Used for Guidance in Prostate Brachytherapy
}

\author{
Andrea Jane Doyle \\ Technological University Dublin, andrea.doyle@tudublin.ie \\ Deirdre King \\ Hermitage Medical Clinic, Dublin, dking@hermitageclinic.ie \\ Jacinta Browne \\ Technological University Dublin, jacinta.browne@tudublin.ie
}

Follow this and additional works at: https://arrow.tudublin.ie/scschphyart

Part of the Physics Commons

\section{Recommended Citation}

Doyle, A.J., King, D.M. \& Browne, J. (2017). A review of the recommendations governing quality assurance of ultrasound systems used for guidance in prostate brachytherapy. Physica Medica, vol. 44, pp.51-57. doi:https://doi.org/10.1016/j.ejmp.2017.11.011

This Article is brought to you for free and open access by the School of Physics \& Clinical \& Optometric Science at ARROW@TU Dublin. It has been accepted for inclusion in Articles by an authorized administrator of ARROW@TU

Dublin. For more information, please contact

arrow.admin@tudublin.ie, aisling.coyne@tudublin.ie, gerard.connolly@tudublin.ie.

Funder: Technological University Dublin (DIT)

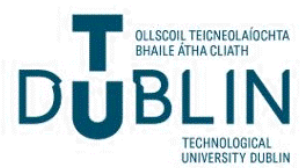




\title{
A Review of the Recommendations Governing Quality Assurance of Ultrasound Systems used for Guidance in Prostate Brachytherapy
}

\author{
Andrea Jane Doyle $e^{a}$ Dr Deirdre M King ${ }^{b}$ and Prof Jacinta E Browne \\ ${ }^{a}$ School of Physics \& Clinical \& Optometric Sciences, Medical Ultrasound Physics and Technology \\ Group, Centre of Industrial Engineering Optics, FOCAS Institute, Dublin Institute of Technology, \\ Ireland \\ ${ }^{b}$ Hermitage Medical Clinic, Dublin, Ireland
}

Author email: andrea.doyle@dit.ie, dking@hermitageclinic.ie, jacinta.bronwe@dit.ie

\section{Abstract}

Ultrasound guided brachytherapy for the treatment of prostate cancer has become a routine treatment option, due to many benefits including patient recovery and dose localisation [1]; however it is not clear whether the standards which govern the image quality for these systems are adequate. Upon review of the recommended standards for ultrasound systems used in prostate brachytherapy procedures, the recommended tests do not appear to be specific to the clinical application of ultrasound guided prostate brachytherapy. Rather they are generic and similar to those recommended for other clinical applications such as general abdominal scanning [2]. Furthermore, there is growing evidence that these tests should be specific to the clinical application [3][4] in order to gain meaningful data about the performance of the system for the application, and also to detect clinically relevant changes in quality control results. An additional problem is that there are no clinically relevant test phantom recommended for the quality assurance of ultrasound systems used in prostate brachytherapy. The image quality for this application of ultrasound 
needs to be monitored to ensure consistent levels of confidence in the procedure. This paper reviews the currently recommended test guidelines and test phantoms for ultrasound systems used in prostate brachytherapy from the different standard bodies and professional organisations. A critical analysis of those tests which are most reflective of the imaging and guidance tasks undertaken in an ultrasound guided prostate brachytherapy procedure will also be presented to inform the design of a TRUS quality assurance protocol.

Key words: Trans-rectal ultrasound (TRUS), quality assurance, image quality, test phantoms, brachytherapy 


\section{Introduction}

Prostate cancer is the second most frequent cause of cancer in European men [5], with similar incidences worldwide. The prognosis for the disease, when diagnosed and managed early, is a three-year survival rate of $94 \%$ [6]. Treatment for the disease is dependent on the malignancy and the stage of the disease. Throughout the management of the disease, diagnostic imaging and in particular, ultrasound imaging play a vital role from diagnosis to medical intervention [6]. Ultrasound imaging is a non-ionizing real-time technique that can provide information about the size, morphological and acoustic characteristics of tumour as well as its application in the guidance of interventional procedures such as a biopsy, treatment planning and treatment delivery. It is clear, that ultrasound is a valuable imaging modality in the treatment of prostate cancer, but it is of paramount importance that reliable and consistent clinical information is provided to adequately treat the patient, and to avoid any complications associated with administering the incorrect dose of radiation, such as repeat procedures, incontinence and radiation proctitis. The importance of quality assurance in prostate brachytherapy centres and the implications of a poor quality brachytherapy service was highlighted by a report that reviewed the performance of prostate brachytherapy in the treatment of prostate cancer during the seven year period of 20022008 in one treatment centre. [7] The report identified many errors, including suboptimal implants resulting in some patients requiring a second intervention and high toxicity causing proctitis in others. The report indicates a lack of training and good quality assurance was responsible for the errors.

Therefore, the ultrasound system needs to be operating optimally and maintained to ensure that any degradation in image quality is detected and not mistaken as a clinical 
anomaly. The quality assurance process should establish the accuracy of the guidance through commissioning and routine testing, detect faults within the scanner with regular quality assurance and should be task specific, relevant to the process of delineating disease.

The guidance and recommendations which are currently available to inform a programme of quality assurance for trans-rectal ultrasound (TRUS) imaging, and the tests phantoms that are available, will be reviewed to determine their efficacy and to inform the design of a TRUS quality assurance protocol.

\section{Brachytherapy}

Ultrasound guided brachytherapy involves the use of (TRUS) imaging to guide the placement of radioactive material into the prostate. There are two methods of brachytherapy that are available for the treatment of prostate cancer; high and low dose rate treatments. The high dose rate treatment can be delivered in a number of fractions or as a complimentary therapy to external beam treatment, and involves the introduction of a high dose radioactive source, usually a wire, into the prostate through a catheter where it is left for a short period of time and removed.. Treatment centres that employ low dose rate brachytherapy implant radioactive material, seeds, into the prostate where they are left to decay to a background level of radioactivity overtime. This type of radiotherapy treatment, similarly to external beam radiotherapy, is planned and pre-treatment imaging is performed using ultrasound imaging. These pre-treatment ultrasound images are used to make volume measurements of the prostate and determine the number of seeds required to deliver the required dose for the procedure. During treatment, volume measurements are also made and visualisation of the prostate and surrounding structures is important for real-time image guidance of the seeds to desired locations for optimal dose delivery. The information acquired from ultrasound images before and during the treatment is used to make 
important clinical decisions and so the value of quality assurance testing of the ultrasound systems used to make these decisions becomes evident.

\section{Quality assurance}

\section{Quality Assurance Guidance and Recommendations}

Professional bodies such as the Royal College of Radiologists and the Society and College of Radiographers recommend ultrasound Quality Assurance (QA) programmes as part of the overall governance of ultrasound examinations [8][9]. The goal of a QA programme for ultrasound systems is to ensure consistent and acceptable levels of system performance, detect faults and document any system degradation over time. The nature of an ultrasound examination means the clinical user makes these decisions in real-time and only a small number of frames are available for review after the decision has been made [10]. A highquality ultrasound procedure requires both a high-quality system and an experienced user. The user is expected to understand the equipment settings and their impact on image quality and, to identify when there are issues with the system and the images produced. Periodic testing is imperative in the assurance of optimum and consistent system quality [11]. Generally, measurements are made and recorded, and baseline values are determined with tolerances and action levels to monitor the systems performance. However, it has been shown that the current QA guidelines may not be relevant to the clinical image quality, and there have been incidences where ultrasound systems have had major faults and were still in clinical use [3][10]. Hangiandreou et al. 2011, found that in a four year study of 45 ultrasound systems, 187 issues were detected with the scanner equipment that was in clinical use [12]. 
The technological advances in ultrasound technology and the increased use of ultrasound as a diagnostic tool, puts an increased demand on the QA programmes. The clinical significance of test parameters will vary with the application. The changes in the imaging parameters need to be related to clinical task and efficacy, and currently the systems of QA do not make this connection [13][3][14]. While there has been work carried out to remove the subjective element of the testing procedures and to automate the process using software $[15][16][17][18]$, the relevance of the QA parameters for each specific application must be considered. Specifically, it is important to link quality assurance parameters with the clinical view of image quality in order to establish what degradation in clinical image quality affects clinical diagnosis or management; this may be achieved through the development of more clinically relevant rest phantoms or task-specific test phantoms.

Quality assurance of ultrasound systems is essential to ensure repeatable and reliable image quality. Professional bodies such as the Institute of Physics and Engineering in Medicine (IPEM), the American institute of Ultrasound in Medicine (AIUM), the European Federation of Societies for Ultrasound in Medicine and Biology (EFSUMB) and the American Association of Physics in Medicine (AAPM), all recommend quality assurance programmes for ultrasound systems to ensure consistent quality of ultrasound images [11][19][20][21]. Each body identifies the need for a testing schedule with regular test intervals, and documentation to monitor and continually assess the equipment performance. While there is some variation in the recommended frequency of the QA tests between the professional bodies, there seem to be at least three distinctive levels of testing that they recommend as illustrated in table 1 [22]. 
The first level of tests "User/Operator tests" are basic intuitive observations to ensure the ultrasound system is operating normally. These tests are quick and provide a good indication of obvious technical issues. The second level of tests "Technical tests" are recommended predominantly for $\mathrm{QA}$ and acceptance purposes. These are tests that are carried out when a new machine is accepted into a department and then at least annually, if not biannually. At acceptance testing, the baseline values are determined and the professional bodies provide guidelines on these values. Then these baselines are considered and tolerances are determined to ensure that at each testing interval the machine is operating at an acceptable level. The "Advanced tests" are not regularly performed; generally only at acceptance testing or after significant repair or changes to the machine. These tests assess characteristics of the system that are very unlikely to drift, and are also monitored through other QA parameters through the periodic testing.

As well as recommended testing schedules, professional bodies such as the AAPM provide guidance on the suitable tolerance levels for ultrasound systems [20], and Task Group 128 [2] also provide details on suitable tolerances for TRUS systems used in prostate brachytherapy. Details of these tolerances are listed in table 2.

Another crucial element of the quality assurance process, are the test phantoms used to assess the image quality. Some QA tests do not require a test phantom such as image uniformity and noise tests [11], but test phantoms are important for a number of other aspects of image quality. There are numerous test phantoms commercially available with general ultrasound QA in mind. These test phantoms are designed with tissue mimicking materials that have been designed to have the same speed of sound as the average of soft 
tissue in the body, $1540 \mathrm{~m} / \mathrm{s}$, and similar attenuation and backscatter values; the recent IPEM Report 102 provides a comprehensive list of the commercially available test phantoms [11]. These test phantoms all have similar generic targets included for annual QA tests, namely the technical tests outlined in Table 1 . These tests phantoms have been designed to assess general image quality parameters and are largely developed with no specific clinical application of ultrasound in mind. While there are commercially available test phantoms that have been constructed to assess the image quality for the ultrasound systems used during brachytherapy procedures, their design is inadequate as they do not include clinically relevant targets to sufficiently test the ultrasound system for this specific application; of prostate brachytherapy [2].

\section{Quality Assurance in Brachytherapy}

There are specific recommendations from the AAPM [2] that outline the parameters that need to be considered specifically for ultrasound guided prostate brachytherapy and suggest some key features for a test phantom for this application. However, like the other recommendations, they are heavily based on the recommendations made for the general application of ultrasound. In TRUS guided brachytherapy the system is used for guidance in intervention and therefore, the quality assurance for this procedure should reflect this application.

The resolution of the Ultrasound system used in prostate brachytherapy system is critical in all three dimensions when identifying the prostate boundary, as well as the implanted seeds and the needle. The guidelines state that axial and lateral resolutions, as well as slice thickness, all affect the resolution, and even though they can be assessed separately it is their overall contribution to the resolution that affects the image [2]. While this is the case 
the guidelines go on to ignore slice thickness and do not recommend evaluating partial volume effects in the testing protocol.

The sensitivity of the system is determined by the depth of penetration, and in particular the depth at which low contrast objects can be detected. The signal-to-noise ratio (SNR) impacts this measurement such that a decrease in the SNR will make it difficult to visualize the prostate [2]. Low contrast detectability is of paramount importance in prostate brachytherapy ultrasound systems. The delineation of the prostate from the surrounding tissue is critical in the area and volume measurements required in the treatment planning for the brachytherapy procedure. It has been shown that for low contrast objects the diameter for threshold detection is dependent on the target contrast, while at higher contrasts the diameter is only weakly dependant on the target contrast [23][15]. Therefore, it is vital to challenge the ultrasound systems contrast detectability at low contrasts and varying sizes.

Area and volume measurements are of particular importance to the brachytherapy procedure as these measurements are involved in the dosimetry calculations, used preoperatively, and during the procedure for the placement of the brachytherapy seeds. To perform this analysis the AAPM guidelines suggest that a spherical test object with known dimensions be used with a moderately high-contrast [2]. Prostate contouring and delineation has been reported to suffer from inter-observer variations on both the TRUS images and on post-operative CT imaging[24]. Lee et al. 2002 presents the results of a case study of ten patients, and found that the ICC of 0.639 for the volume of the prostate was good but the two dosimetric quantifiers $D_{90}$ the minimal dose received by $90 \%$ of prostate, and $V_{100}$ the percentage of prostate volume receiving $100 \%$ of prescribed minimal 
peripheral dose, to be poor at ICC 0.275 and ICC 0.345 respectively[25]. The prostate's contrast in an ultrasound image makes it difficult to outline the gland from the surrounding tissue[2]. Sandhu et al. demonstrated an improvement in prostate volume using tissue harmonic imagining compared to conventional B-mode imagining in both a phantom and a patient study [26][27]. Improvements in tissue differentiation that were associated with tissue harmonic imaging improved the prostate contour and thereby, the corresponding volume estimates. Therefore, an object such as that described by the AAPM would not sufficiently challenge the ultrasound system and test its capabilities for the prostate brachytherapy application. The accuracy of the volume measurement and the ability to delineate a poorly defined border should be addressed independently.

Another unique feature to the application of ultrasound in the prostate brachytherapy procedure is the grid and stepper system. The TRUS probe is placed in a rig mounted with a stepper that is manually manipulated to introduce the probe into the rectum in specific increments. The grid is used to align the needles, which are used to introduce the seeds into the prostate, to specific locations represented on the ultrasound system. Ensuring that the grid is aligned to the grid displayed on the image is imperative in the successful treatment of patients; the seeds are distributed inside the prostate to ensure optimal dose distribution to the prostate while sparing organs at risk such as the bladder and the rectum.

\section{Test phantoms}

Quality assurance test phantoms are an important part of an extensive and effective QA programme. A good test phantom should be clinically relevant and be designed to assess the ultrasound system in the context of the clinical application, and furthermore, be capable of detecting issues before they have a clinical impact. Image quality refers to the aspects of 
the image which are expected to impact the diagnostic capability of the system [11]. The factors that affect the image quality and diagnosis for one application will differ to another, and it is important to identify these factors and design a test phantom to ensure that these image quality parameters are examined [3][28][29]. There are several test phantoms commercially available to assess image quality, as well as specific test phantoms designed for individual applications. Key features of a test phantom include clinically relevant targets and contrasts. Some of the most common targets that are included in test phantoms are targets at specific depths and spacing that can be used to assess the distance calibration and the spatial resolution. However, the majority of these test phantoms do not include targets for slice thickness assessment therefore, artefacts such as the partial volume effect cannot be evaluated which impacts needle tip visualisation (Ref). A homogenous known material such as the background of a test phantom can be used to assess the image uniformity and the sensitivity of the system. Some test phantoms also include targets of varying backscatter values and sizes to assess the contrast detectability. These are the basic targets included in a test phantom used to assess image quality for the general application of diagnostic ultrasound. However, as ultrasound imaging has moved into many diagnostic, and some interventional fields, it is important to assess the efficacy of the test parameters for specific clinical applications. It is important to identify the technical measures which will have the best correlation with clinically relevant parameters of quality and thus, the greatest impact on clinical outcomes and which pose the greatest risk if they change. A further consideration is whether it is possible to measure all clinical measures of quality directly and if not, how can this risk be controlled in the QA process. The growth of technologies has surpassed the production of new test phantoms and test protocols, and it is important to be 
proactive in the management of QA programmes to ensure that equipment is robustly interrogated to guarantee that systems are operating at an optimum level of quality [10].

Table 3 presents the suppliers of quality assurance test phantoms, with details of the specific models that are compatible a TRUS probe.

There have also been a number of test phantoms developed by research groups for quality assurance of trans-rectal ultrasound systems, for the evaluation of specific parameters; from volume and geometry accuracy to more complex image artefacts that may cause inaccurate seed placement and sub optimal treatment outcomes.

The volume of the prostate is an important factor in treatment planning for dose determination and distribution. Drever et al. 2007 describes a simple agarose based test phantom developed for volume measurements that can be used for routine daily QA of volume accuracy. The nature of the brachytherapy procedure requires accurate needle insertion and seed placement to ensure optimal dose delivery and treatment outcomes. Goldstein et al. 2002 [30] designed a simple yet effective test phantom of ethylene and glycerol, that can be used to validate each TRUS probe to ensure that the needle grid used during the procedure and the overlay grid displayed on the ultrasound system, are correctly aligned. Ryu et al. 2012 [31] describes a custom-built agar based test phantom, designed from clinical CT prostate data, used to examine seed placement accuracy, in particular for larger prostates. During the prostate brachytherapy procedure, the needles are inserted into the prostate perpendicular to the transverse ultrasound beam and as such there can be 
errors in needle tip localisation and seed placement, as well as side lobes that can cause further issues with localisation. Peikari et al.2012 [32] descried a Wire-Bridge test phantom that allows for elevation beamwidth measurements to examine the uncertainties associated with localisation. In a study by Sandhu et al. 2010 [26], the improvements of tissue harmonic imagining over B-mode imagining for the application of ultrasound guided prostate brachytherapy were investigated for a number of the system parameters resolution, geometric accuracy, depth of penetration, dead zone, signal-to-noise ratio and tissue-toclutter ratio, to compare the two modes using an in house test phantom made from homogenized milk based Tissue mimicking material. As the patient pathway for prostate cancer treatment usually requires a multimodality approach to the diagnostic imaging employed it is advantageous to have a test phantom that can be used for cross modality validation of all of the diagnostic imaging employed. Hungr et al. 2012 described a test phantom that can be used with TRUS CT and MRI that also exhibits the deformability characteristics of tissue.

\section{Phantoms for TRUS Brachytherapy}

Presented in figure 1 are some of the commercially available test phantoms recommended for quality assurance of TRUS systems. Although some test phantoms have evolved and designed a cavity to insert the TRUS probe, they have not been modified to include clinically applicable test objects of relevant size, depth or contrast.

Recommendations from the AAPM task group report 128 [2] include some important design elements for a TRUS test phantom, such as a prostate target and the clinically relevant 
orientation. However, this report recommends a high contrast prostate target relevant to background and this does not mimic the clinical situation. A high contrast target is available in a currently available CIRS training test phantom, but as shown in figure 2 the contrast is not applicable. The contrast resolution is vital in the TRUS imaging performed during brachytherapy; the delineation of the prostate, and therefore the ability to accurately assess the volume is critical to the procedure.

A suitable test phantom to be used in Ultrasound imaging will have a tissue mimicking material (TMM) with acoustic properties and mechanical properties of the tissue as close to that of soft tissue. Investigations into suitable TMM for US began in the late 1970's using gel and served as a platform for future work [33]. The use of organic materials such as animal tissues and foodstuffs as suitable TMM, have also been investigated. The TMM acoustic properties need to be as close to that of the relevant soft tissue as possible. There are 3 essential acoustic parameters of a successful TMM; speed of sound (SOS), attenuation coefficient and backscatter [34]. The acoustic properties of prostate tissue have been reported in the literature with attenuation value of $1.86 \pm 0.14 \mathrm{~dB} / \mathrm{mm}$ and acoustic velocity of $1614 \pm 30 \mathrm{~m} / \mathrm{s}$ in normal prostate and $1.42 \pm 0.08 \mathrm{~dB} / \mathrm{mm}$ and $1584 \pm 12 \mathrm{~m} / \mathrm{s}$ in prostate cancer respectively [35]. International bodies such as the IEC and the AIUM have specified the acoustic velocity $1540 \mathrm{~m} / \mathrm{s}$ and attenuation as $0.5 \& 0.7 \mathrm{~dB} / \mathrm{cm} / \mathrm{Mhz}$. The advantages and disadvantages of a range of tissue mimicking materials are discussed in the literature; in relation to their acoustic properties and suitability to specific applications [36]. Table 4, adapted from Culjat et al.[36] and Browne et al. [37], presents the acoustic velocity and attenuation for a number of these materials. 
The materials that are in the commercially manufactured test phantoms and the IEC Agar TMM [47] which can be produced for custom and research phantoms, have been investigated and acoustically characterised to determine their stability and limitations in the context of quality assurance testing [37]. Browne et al. investigated the effects of temperature and frequency dependency of the materials. These are important considerations for a quality assurance phantom; a test phantom should be stable and only changes in the ultrasound system performance should be highlighted at the quality assurance testing. Changes in the acoustic properties, specifically the velocity, can cause distortion in distance measurements and this has the potential to effect resolution measurements [48][49].

As well as the acoustic properties, the mechanical properties of a TMM should be considered when designing a suitable test phantom. Professional bodies do not recommend specific mechanical properties for suitable TMM in quality assurance test phantoms. Shear wave elastography is an ultrasound imaging technique that aims to quantitatively image a tissue to determine the stiffness, as differences in the mechanical properties, such as the compressibility, can be an indication of disease in tissue [50]. This property of the tissue is the Young's modulus and indicates the stiffness if the tissue. It indicates the relationship between stress and strain, and is a pressure measured in $\mathrm{kPa}$. In the prostate, it has been shown that cancerous tissue has a greater Young's modulus than that of healthy tissue [51][52][53]; indicating that diseased tissue is stiffer than that of healthy tissue. This type of stiffness can be matched in TMM by varying the concentration of the components in the material. Cournane et al. [54] reviewed quality control test phantoms for ultrasound 
elastography and proposed mechanical properties for TMM to mimic various tissues in the body. The proposed range for prostate tissue was $15 \mathrm{kPa}$, with malignancy between 10-15 $\mathrm{kPa}$.

The longevity of the material used in a test phantom also needs to be considered. Economically it is favourable for a test phantom to remain stable for a long period of time. The shelf life of a phantom should be clear and should be considered when acquiring a test phantom. Imaging a test phantom, before quality assurance tests are performed, using another modality such as $\mathrm{CT}$, can be a convenient method to examine any changes in the phantom itself. Another indication of degradation is the weight of the test phantom. The stability of the test phantom overtime will be important in consistency in quality assurance testing; deterioration should be due to US system performance and not degradation of the test phantom.

There are many factors that need to be considered in the design of a suitable test phantom for the application of TRUS imaging for the application of brachytherapy. The targets included should be designed to assess all aspects of the image quality, and the test phantom needs to be constructed to effectively assess each transducer view used during the procedure.

\section{Discussion}

the American Association of Physics in Medicine (AAPM) [2] offer guidance and specific recommendations for quality assurance of TRUS systems used in brachytherapy procedures. However, it is important to consider if each of the conventional test parameters assess relevant features of image quality of a TRUS image and how to conduct the tests to ensure that any degradation in the image quality is detected. The test parameters and test 
phantoms have been presented and will now be discussed in terms of their relevance and efficacy to this application of TRUS image quality.

The resolution of a TRUS image for prostate brachytherapy is important for boundary detection, however the AAPM do not provide any recommendations for the measurement of the slice thickness. The partial volume effect has the potential to cause misdiagnosis, where cystic structures are not represented in the image due to an accumulation of scan lines when the beam width is reduced. This can cause fill-in of the cyst, either partially or totally in the image [55]. Small spherical lesions with varying contrast could be incorporated into a clinically applicable test phantom. These "cysts" could be used to assess the slice thickness as well as the axial and lateral resolution and could also be used to determine the contrast detectability of the system if targets of varying contrast were included.

The sensitivity of the system will impact the visualization the prostate [2], and the ability of the user to delineate the prostate from surrounding tissue. Assessing the low contrast detectability of the system will allow for a relevant measure of the sensitivity of a system used in TRUS brachytherapy. This test design should be considered for both the methodology of the test procedure and the design of the test object. Targets with small variations in contrast, which are clinically relevant at assorted sizes, would be ideal targets in a brachytherapy test phantom to assess ultrasound image quality.

A prostate mimicking target is of paramount importance in the design of a test phantom for a prostate brachytherapy ultrasound test phantom. A low contrast prostate target of known, clinically applicable dimensions could be used to assess area and volume measurements. 
The grid that is used during the procedure to align the needles that introduce the sources into the prostate under TRUS image guidance should also be evaluated. The physical alignment tool and electronic grid alignment should be verified with a suitable set of targets in the test phantom; a grid of moderate to high contrast targets that can verify the spatial accuracy of the alignment.

A test phantom for this application of ultrasound should be designed with the prostate brachytherapy procedure in mind, considering probe orientation and operation, with attention to include both sagittal and transverse views. The TRUS probe should be introduced into the test phantom through a cavity in the appropriate configuration, i.e. the test phantom should be above the probe. The depths of the structures and targets inside the test phantom are an important consideration. Clinically the configuration of the probe in the rectal cavity gives an optimum imaging location; with only a few millimetres of rectal wall separating the probe from the cavity. This should be emulated in a test phantom when designing and positioning prostate targets. Both views on the probe are used during the procedure, at both biopsy and interventional stages. The sagittal array is used to assess the length of the prostate and the transverse array is used to assess the width and height of the gland. This can be problematic with traditional test phantoms that generally incorporate an imaging plane designed for linear array transducer, and can impede the evaluation of the transverse field of view. A test phantom with an imagining window or probe holder that will allow for orientation of the probe to examine the image quality of both sagittal and transverse transducer arrays in a reliable manner would facilitate a more reproducible quality assurance programme. 
Quality assurance recommendations for a testing protocol and an application specific test phantom for the ultrasound systems used in TRUS guided prostate brachytherapy are presented in table 5. 


\section{Conclusion}

Trans-rectal ultrasound is a valuable tool in the diagnosis and treatment of prostate cancer. It is important to monitor system performance and perform regular quality assurance that is specific to this application. This includes a regime of tests that are designed to capture indicators of image quality degradation, and a test phantom that can effectively detect these adverse anomalies in the image. The design should be suitable for the end user in a hospital environment without compromising the clinical relevance.

\section{Acknowledgements}

The authors would like to acknowledge Dublin Institute of Technology Dean of Graduate Fiosraigh Research Programme for funding this study. 


\section{References}

[1] D. G. Petereit et al., "Brachytherapy: Where has it gone?," J. Clin. Oncol., vol. 33, no. 9, pp. 980-982, 2015.

[2] D. Pfeiffer, S. Sutlief, W. Feng, H. M. Pierce, and J. Kofler, "AAPM Task Group 128: quality assurance tests for prostate brachytherapy ultrasound systems.," Med. Phys., vol. 35, no. 12, pp. 5471-5489, 2008.

[3] J. E. Browne, "An Investigation of the Relationship Between in Vitro and in Vivo Ultrasound Image Quality," Ultrasound, vol. 12, no. 4, 2004.

[4] L. Cannon, "Development of Quality Assurance and Anthropomorphic Phantoms for Breast Ultrasound Imaging," Dublin Institute of Technology, Kevin Street, Dublin 8, 2013.

[5] J. Ramon and L. J. Denis, Prostate Cancer (Recent Results in Cancer Research, Vol. 175). Berlin Heidelberg: Springer-Verlag, 2007.

[6] National Cancer Registry, "Cancer in Ireland 1994-2013: Annual Report of the National Cancer Registry.," Cork, Ireland., 2015.

[7] Department of Veterans Affairs Office of Inspector General, "Health Inspection: Review of Brachytherapy Treatment of Prostate Caner, Philadelphia, Pennsylvania and Other VA Medical Centres. Report No. 09-02815-143.," Washington DC, 2010.

[8] P. Dubbins, "Royal College of Radiologists Standards for Ultrasound," London, 2005.

[9] BMUS, “Guidelines For Professional Ultrasound Practice SOCIETY AND COLLEGE OF RADIOGRAPHERS AND BRITISH MEDICAL," no. December 2015, 2015.

[10] S. Russell, "Ultrasound quality assurance and equipment governance," Ultrasound, vol. 22, no. 1, pp. 66-69, Dec. 2014.

[11] S. Russell, Ed., IPEM Report no 102 Quality Assurance of Ultrasound Imaging Systems. York, 
UK: Institute of Physics and Engineering in Medicine, 2010.

[12] N. J. Hangiandreou, S. F. Stekel, D. J. Tradup, K. R. Gorny, and D. M. King, "Four-Year Experience with a Clinical Ultrasound Quality Control Program," Ultrasound Med. Biol., vol. 37, pp. 1350-1357, 2011.

[13] N. J. Dudley, K. Griffith, G. Houldsworth, M. Holloway, and M. A. Dunn, "A review of two alternative ultrasound quality assurance programmes," Eur. J. Ultrasound, vol. 12, pp. 233245, 2001.

[14] S. Wolstenhulme et al., "Agreement between objective and subjective assessment of image quality in ultrasound abdominal aortic aneurism screening," Br. J. Radiol., vol. 88, pp. 1-9, 2014.

[15] S. W. Smith, R. F. Wagner, J. M. Sandrik, and H. Lopez, "Low Contrast Detectability and Contrast/Detail Analysis in Medical Ultrasound," IEEE Trans. Sonics Ultrason., vol. 30, no. 3, pp. $164-173,1983$.

[16] N. M. Gibson, N. J. Dudley, and K. Griffith, "A computerised quality control testing system for B-mode ultrasound," Ultrasound Med. Biol., vol. 27, no. 12, pp. 1697-1711, 2001.

[17] J. E. Browne, A. J. Watson, N. M. Gibson, N. J. Dudley, and A. T. Elliott, “Objective measurements of image quality," Ultrasound Med. Biol., vol. 30, no. 2, pp. 229-237, 2004.

[18] J. M. Kofler, M. J. Lindstrom, F. Kelcz, and E. L. Madsen, “Association of automated and human observer lesion detecting ability using phantoms," Ultrasound Med. Biol., vol. 31, no. 3, pp. 351-359, 2005.

[19] J. A. . Boote, Evan J.; Forsberg, Flemming; Garra, Brian S.; Ophir, Johathan; Ophir, Karen J.; Zagzebski, AIUM Routine Quality Assurance for Diagnostic Ultrasound Equipment. Maryland, USA: American Institute of Ultrasound in Medicine, 2008. 
[20] M. M. Goodsitt, P. L. Carson, S. Witt, D. L. Hykes, and J. M. Kofler, "Real-time B-mode ultrasound quality control test procedures. Report of AAPM Ultrasound Task Group No. 1.," Med. Phys., vol. 25, no. 8, pp. 1385-1406, 1998.

[21] C. Kollmann, C. DeKorte, N. J. Dudley, N. Gritzmann, K. Martin, and D. H. Evans, "Guideline for Technical Quality Assurance (TQA) of ultrasound devices (B-Mode)--version 1.0 (July 2012): EFSUMB Technical Quality Assurance Group--US-TQA/B.," Ultraschall Med., vol. 33, no. 6, pp. 544-9, Dec. 2012.

[22] A. J. Doyle and J. E. Browne, "Review of the guidance governing quality assurance of ultrasound systems used for guidance in prostate brachytherapy," Phys. Medica, vol. 32, no. 7, pp. 953-954, Jul. 2016.

[23] S. W. Smith and H. Lopez, "A contrast-detail analysis of diagnostic ultrasound imaging," Med. Phys., vol. 9, no. 1, pp. 4-12, 1982.

[24] M. C. Solhjem et al., "Prostate volume measurement by transrectal ultrasound and computed tomography before and after permanent prostate brachytherapy," Int. J. Radiat. Oncol. Biol. Phys., vol. 60, no. 3, pp. 767-776, 2004.

[25] W. R. Lee, M. Roach, J. Michalski, B. Moran, and D. Beyer, "Interobserver variability leads to significant differences in quantifiers of prostate implant adequacy," Int. J. Radiat. Oncol. Biol. Phys., vol. 54, no. 2, pp. 457-461, 2002.

[26] G. K. Sandhu, P. B. Dunscombe, and R. F. H. Khan, "A pre-clinical phantom comparison of tissue harmonic and brightness mode imaging for application in ultrasound guided prostate brachytherapy," Phys. Medica, vol. 27, no. 3, pp. 153-162, 2011.

[27] G. K. Sandhu, P. Dunscombe, T. Meyer, S. Pavamani, and R. Khan, "Inter- and intra-observer variability in prostate definition with tissue harmonic and brightness mode imaging," Int. J. Radiat. Oncol. Biol. Phys., vol. 82, no. 1, pp. 9-16, 2012. 
[28] S. C. Metcalfe and J. A. Evans, "A study of the relationship between routine ultrasound quality assurance parameters and subjective operator image assessment," Br. J. Radiol., vol. 65, no. 775, pp. 570-575, Jul. 1992.

[29] L. Beales, S. Wolstenhulme, J. A. Evans, R. West, and D. J. A. Scott, "Reproducibility of ultrasound measurement of the abdominal aorta," Br. J. Surg., vol. 98, no. 11, pp. 1517-1525, 2011.

[30] A. Goldstein, M. Yudelev, R. K. Sharma, and E. Arterbery, "Design of quality assurance for sonographic prostate brachytherapy needle guides.," J. Ultrasound Med., vol. 21, no. 9, pp. 947-54, Sep. 2002.

[31] B. Ryu et al., "Prostate Brachytherapy With Oblique Needles to Treat Large Glands and Overcome Pubic Arch Interference," Int. J. Radiat. Oncol., vol. 83, no. 5, pp. 1463-1472, Aug. 2012.

[32] M. Peikari, T. K. Chen, A. Lasso, T. Heffter, G. Fichtinger, and E. C. Burdette, "Characterization of ultrasound elevation beamwidth artifacts for prostate brachytherapy needle insertion," Med. Phys., vol. 39, no. 1, p. 246, 2012.

[33] E. L. Madsen, J. a Zagzebski, R. a Banjavie, and R. E. Jutila, "Tissue mimicking materials for ultrasound phantoms.," Med. Phys., vol. 5, no. 5, pp. 391-394, 1978.

[34] L. A. DeWerd and M. Kissick, Eds., The Phantoms of Medical and Health Physics. New York, NY: Springer New York, 2014.

[35] H. Tanoue, Y. Hagiwara, K. Kobayashi, and Y. Saijo, "Ultrasonic tissue characterization of prostate biopsy tissues by ultrasound speed microscope," Proc. Annu. Int. Conf. IEEE Eng. Med. Biol. Soc. EMBS, pp. 8499-8502, 2011.

[36] M. O. Culjat, D. Goldenberg, P. Tewari, and R. S. Singh, "A review of tissue substitutes for ultrasound imaging," Ultrasound Med. Biol., vol. 36, no. 6, pp. 861-873, 2010. 
[37] J. E. Browne, K. V. Ramnarine, A. J. Watson, and P. R. Hoskins, "Assessment of the acoustic properties of common tissue-mimicking test phantoms," Ultrasound Med. Biol., vol. 29, no. 7, pp. 1053-1060, 2003.

[38] M. M. Burlew, E. L. Madsen, J. a Zagzebski, R. a Banjavic, and S. W. Sum, “A new ultrasound tissue-equivalent material.," Radiology, vol. 134, no. 2, pp. 517-520, 1980.

[39] E. L. Madsen, G. R. Frank, and F. Dong, "Liquid or solid ultrasonically tissue-mimicking materials with very low scatter," Ultrasound Med. Biol., vol. 24, no. 4, pp. 535-542, 1998.

[40] K. V Ramnarine, T. Anderson, and P. R. Hoskins, "Construction and geometric stability of physiological flow rate wall-less stenosis phantoms," Ultrasound Med. Biol., vol. 27, no. 2, pp. 245-250, Feb. 2001.

[41] W. D. D’Souza, E. L. Madsen, O. Unal, K. K. Vigen, G. R. Frank, and B. R. Thomadsen, "Tissue mimicking materials for a multi-imaging modality prostate phantom.," Med. Phys., vol. 28, no. 4, pp. 688-700, 2001.

[42] N. L. Bush and C. R. Hill, "Gelatine-alginate complex gel: a new acoustically tissue-equivalent material.," Ultrasound Med. Biol., vol. 9, no. 5, pp. 479-84.

[43] A. Kharine et al., "Poly(vinyl alcohol) gels for use as tissue phantoms in photoacoustic mammography.," Phys. Med. Biol., vol. 48, no. 3, pp. 357-70, Feb. 2003.

[44] T. Kondo, M. Kitatuji, and H. Kanda, "Newtissue mimicking materials for ultra- sound phantoms," in Ultrasonics Symposium 2005 IEEE, 2005, pp. 1664-1667.

[45] G. Wojcik, T. Szabo, J. Mould, L. Carcione, and F. Clougherty, "Nonlinear pulse calculations and data in water and a tissue mimic," in Ultrasonics Symposium, Proceedings IEEE, 1999, pp. $1521-1526$.

[46] A. Giacomini, "Ultrasonic velocity in ethanol-water mixtures," J. Acoust. Soc. Am., vol. 19, pp. 
701-702, 1974.

[47] C. Teirlinck, R. Bezemer, KollmannC, and E. Al., "Development of an example flow test object and comparison of five of these objects, constructed in various laboratories.," Ultrasonics, vol. 36, pp. 653-660, 1998.

[48] A. Goldstein, "THE EFFECT OF ACOUSTIC VELOCITY ON PHANTOM MEASUREMENTS," Ultrasound Med. Biol., vol. 26, no. 7, pp. 1133-1143, 2000.

[49] N. J. Dudley, N. M. Gibson, M. J. Fleckney, and P. D. Clark, "The effect of speed of sound in ultrasound test objects on lateral resolution," Ultrasound Med. Biol., vol. 28, no. 11-12, pp. 1561-1564, 2002.

[50] J. L. Gennisson, T. Deffieux, M. Fink, and M. Tanter, "Ultrasound elastography: Principles and techniques," Diagn. Interv. Imaging, vol. 94, no. 5, pp. 487-495, 2013.

[51] B.-M. Ahn, J. Kim, L. Ian, K.-H. Rha, and H.-J. Kim, "Mechanical Property Characterization of Prostate Cancer Using a Minimally Motorized Indenter in an Ex Vivo Indentation Experiment," J. Urol., vol. 76, no. 4, pp. 1007-1011, 2010.

[52] W. C. Carson, G. J. Gerling, T. L. Krupski, C. G. Kowalik, J. C. Harper, and C. A. Moskaluk, "Material characterization of ex vivo prostate tissue via spherical indentation in the clinic," Med. Eng. Phys., vol. 33, pp. 302-309, 2010.

[53] R. Cao, Z. Huang, T. Varghese, and G. Nabi, "Tissue mimicking materials for the detection of prostate cancer using shear wave elastography: A validation study," Med. Phys., vol. 40, no. 2, p. 22903, 2013.

[54] S. Cournane, A. Fagan, J. Browne, A. J. Fagan, and J. E. Browne, "Review of Ultrasound Elastography Quality Control and Training Test Phantoms," Ultrasound, vol. 20, pp. 1-2, 2012.

[55] W. R. Hendrick, D. L. Hykes, and D. E. Strachman, Ultrasound Physics and Instrumentation, 
Third. Missouri: Mosby, 1995. 